\title{
Ablación de arrritmias: resultados actualmente reproducibles
}

\author{
Dr. Mauricio Moreno
}

Recibido el 23 de julio de 2009. Aceptado el 25 julio de 2009

Rev Chil Cardiol 2009; 28: 211-213

El estudio electrofisiológico más ablación constituye hoy en día una alternativa efectiva y segura para el tratamiento de las arritmias. Los primeros reportes sobre terapia con fulguración o ablación de arritmias se concentraban esencialmente en las taquicardias supraventriculares y, en especial, en el síndrome de Wolf Parkinson White (WPW). Hoy en día prácticamente todas las arritmias, tanto supraventriculares como ventriculares, tienen una opción de tratamiento con la ablación, inclusive arritmias tan complejas como la fibrilación auricular ${ }^{1}$ y fibrilación ventricular2.

En el artículo de Kuzmicic et $\mathrm{al}^{3}$ aparecido en este número de la revista, describen los resultados de 11 años de experiencia de actividad electrofisiológica en un centro, con resultados comparables a la literatura internacional. Kusmicic et al, reportan resultados exitosos para la ablación de taquicardia intranodal del 93\%, para las vías accesorias de $92 \%$ y para el flutter auricular común de $93 \%$. El registro de ablación del NASPE reportó tasas de éxito de ablación para la taquicardia intranodal de $96 \%{ }^{4}$, los reportes de ablación exitosa de vías accesorias es del $95 \% 5 y$ el éxito de la ablación del flutter auricular varía entre 90 a $100 \%{ }^{6}$. Como se señaló, son cifras comparables y acreditan que la ablación es un tratamiento efectivo y especialmente reproducible hoy en día.

Los resultados tan favorables de la ablación son consecuencia de la experiencia y adquisición de nuevos conceptos electrofisiológicos. Por ejemplo, inicialmente la ablación del flutter auricular común consistía en realizar líneas de ablación sobre el istmo cavotricuspídeo, sitio crítico en la mantención del circuito reentrante; si la arritmia cedía durante la ablación el procedimiento se consideraba exitoso.
Pero con esta estrategia se observó una alta tasa de recurrencia del flutter auricular 7 . Posteriormente se exigió no sólo eliminar el flutter sino también demostrar bloqueo bidireccional del istmo cavotricuspídeo, que consiste en la ausencia de conducción eléctrica a través de este istmo, tanto en sentido horario como antihorario. Este requisito electrofisiológico optimizó los resultados y consolidó a la ablación como la terapia de elección para el flutter auricular común.

La taquicardia paroxística supraventricular más frecuente es la taquicardia por reentrada intranodal, consecuentemente es una de las taquicardias que con más frecuencia se aborda para ablación. Al igual que en el flutter auricular común, en la taquicardia intranodal el sitio anatómico para la ablación es conocido e igual en cada paciente (no hay necesidad del mapear el foco de la arritmia). El circuito reentrante de la taquicardia intranodal está ubicado cerca del nodo aurículo-ventricular (AV); cuando la taquicardia es típica, el brazo anterógrado del circuito es la vía nodal lenta, mientras que el brazo retrógrado es la vía nodal rápida. Aunque la proximidad del nodo AV constituye un riesgo para la ablación de inducir bloqueo AV, el actual abordaje de ablación sobre la vía nodal lenta ha permitido reducir este riesgo a menos de el $1 \% 4$. Este concepto de seguridad asociado a la ablación ha permitido incrementar las indicaciones de esta terapia.

Actualmente se considera a la ablación como la terapia de primera línea para el síndrome de WPW sintomático. En este caso hay una vía accesoria que conecta eléctricamente las aurículas y los ventrículos independiente del nodo AV. Cuando esta vía puede conducir en dirección retrógrada (desde ventrículo a aurícula) el paciente puede desarrollar taquicardias

Correspondencia: Dr. Mauricio Moreno

Clínica Dávila

Correo Electrónico:mauricio.moreno@davila.cl 
paroxísticas supraventriculares con complejo QRS estrecho, llamada taquicardia tipo ortodrómica, donde el brazo anterógrado de la reentrada es el nodo AV y el brazo retrógrado es la vía accesoria. Además, las vías accesorias pueden conducir en dirección anterógrada (desde aurícula a ventrículo), con la posibilidad de establecer taquicardias reentrantes con complejo QRS ancho llamadas taquicardias antidrómicas (el brazo anterógrado del circuito es la vía accesoria y el brazo retrógrado es el nodo AV). También, estos pacientes cuando se presenta una fibrilación auricular (FA) tienen el riesgo de conducción rápida al ventrículo con respuestas ventriculares muy elevadas que pueden degenerar en fibrilación ventricular. Si bien es cierto, está arritmia se observa sólo entre 0.15 a $0.39 \%$ de los pacientes con WPW, 8 dado que su ocurrencia es catastrófica, esto determina que, incluso en pacientes con WPW asintomático, la ablación pueda considerarse como el tratamiento de elección.

La adquisición de nuevos conceptos sobre la fisiopatología de la FA ha permitido abordar su tratamiento en forma curativa. En el año 1998 el Dr. Haissaguerre ${ }^{1}$ reportó un novedoso estudio de ablación de la fibrilación auricular, que consistió esencialmente en el aislamiento eléctrico de las venas pulmonares. Las venas pulmonares son el foco de origen de la FA en el $90 \%$ de los casos; otros posibles focos son la vena cava superior, ligamento de Marshall, etc. Por ello la mayoría de las estrategias de ablación de la FA hoy en día se centran fundamentalmente en aislar eléctricamente las venas pulmonares. El éxito de la ablación de FA, según se ha reportado, es aproximadamente del $70 \%$ a $80 \%$; estos resultados dependen en gran medida de la selección de los pacientes $^{9}$ La posibilidad de recurrencia es del $20 \%$, generalmente debida a la reconducción eléctrica de las venas pulmonares ablacionadas y menos frecuente es la aparición de nuevos focos de FA no ablacionados. Las complicaciones asociadas a este procedimiento se han reducido gracias a ajustes en la técnica, como evitar las aplicaciones de radiofrecuencia dentro de las venas pulmonares, con lo cual se ha disminuido a menos de el $1 \%$ el riesgo de estenosis de las venas pulmonares 9 . También se han reducido los riesgos con la introducción de nuevas tecnologías que permiten mejor apoyo anatómico y eléctrico durante el procedimiento.

En este sentido el principal nuevo avance tecnológico es la incorporación de los sistemas de navagación tridimensional. Los más usados universalmente y disponibles en nuestro medio son el sistema ENSITE y el sistema CARTO. Estos sistemas permiten entregar información anatómica, complementaria a la radioscopía utilizada tradicionalmente en estos procedimientos, e información eléctrica de las arritmias estudiadas. Esta información eléctrica se divide básicamente en información en los tiempos de conducción (mapa de activación) e información sobre los voltajes eléctricos (mapa de voltaje). A través de esta información es posible abordar con mayor precisión arritmias focales (por ejemplo, taquicardia auricular) y arritmias reentrantes (por ejemplo, taquicardia ventricular debido a una cicatriz de infarto o flutter auricular atípico).

Otras tecnologías incorporadas a la electrofisiología son la ecografía intracardíaca como apoyo anatómico, nuevas fuentes de energía de ablación (crioablación, láser, etc), estereotaxia y robótica. Pero todos estas tecnologías requieren necesariamente de un acabado conocimiento de la electrofisiología básica para su adecuada aplicación en los procedimientos diagnósticos y de ablación.

Por lo tanto, los procedimientos electrofisiológicos son un tratamiento efectivo y seguro para las arritmias, basado en los conceptos electrofisiológicos básicos y en las nuevas tecnologías incorporadas. La reproducibilidad de los resultados entre los diferentes centros es un elemento muy importante, y que se ratifica favorablemente en los resultados publicados en el artículo de Kuzmicic et al3.

\section{Referencias}

1. HAÏSSAGUERRE M, JAÏS P, SHAH DC, et al. Spontaneous initiation of atrial fibrillation by ectopic beats originating in the pulmonary veins. N Engl J Med. 1998; 339: 659 - 666.
2. WRIGHT M, SACHER F, HAÏSSAGUERRE M. Catheter ablation for patients with ventricular fibrillation. Curr Opin Cardiol. 2009; 24: 56 - 60. 
3. KUZMICIC B, CASTRO G, GOMEZ F, ESPINOZA C . Estudios electrofisiológicos, efectividad real de ablaciones en arritmias y sus complicaciones en Antofagasta, como centro de derivación de la segunda región de Chile. Rev Chil Cardiol 2009; 28: 185-192

4. SCHEINMAN MM, HUANG S. The 1998 NASPE prospective catheter ablation registry. Pacing Clin Electrophysiol 2000; 23: 1020 - 1028.

5. CALKINS H, YONG P, MILLER JM, et al, for the Atakr Multicenter Investigators Group. Catheter ablation of accessory pathways, atrioventricular nodal reentrant tachycardia, and the atrioventricular junction: final results of a prospective, multicenter clinical trial. Circulation 1999; 99 : 262 - 270.

6. CHEN SA, CHIANG CE, WU TJ, et al. Radiofrequency catheter ablation of common atrial flutter:comparison of electrophysiologically guided focal ablation technique and linear ablation technique. J Am Coll Cardiol 1996; 27: $860-868$

7. SAXON LA, KALMAN JM, OLGIN JE, SCHEINMAN MM, LEE RJ, LESH MD. Results of radiofrequency catheter ablation for atrial flutter Am J Cardiol 1996; 77: 1014 - 1016.

8. MUNGER TM, PACKER DL, HAMMILL SC, et al. A population study of the natural history of Wolff-Parkinson-White syndrome in Olmsted County, Minnesota, 1953-1989. Circulation 1993; 87: 866-73.

9. CALKINS H, BRUGADA J, PACKER DL, et al. HRS/EHRA/ECAS expert consensus statment on catheter and surgical ablation of atrial fibrillation: recomendations for personnel, policy, procederes and follow-up. A report of the Heart Rhythm Society (HRS) Task Force on catheter and surgical ablation of atrial fibrillation. Heart Rhythm 2007; 4: 816 - 861. 